\title{
Video Article \\ Interictal High Frequency Oscillations Detected with Simultaneous Magnetoencephalography and Electroencephalography as Biomarker of Pediatric Epilepsy
}

\author{
Christos Papadelis ${ }^{1}$, Eleonora Tamilia ${ }^{1}$, Steven Stufflebeam ${ }^{2}$, Patricia E. Grant ${ }^{1}$, Joseph R. Madsen ${ }^{3}$, Phillip L. Pearl ${ }^{4}$, Naoaki Tanaka ${ }^{2}$ \\ ${ }^{1}$ Fetal-Neonatal Neuroimaging and Developmental Science Center, Division of Newborn Medicine, Department of Medicine, Boston Children's Hospital, Harvard Medical \\ School \\ ${ }^{2}$ Athinoula A. Martinos Center for Biomedical Imaging, Massachusetts General Hospital, Harvard Medical School \\ ${ }^{3}$ Division of Epilepsy Surgery, Department of Neurosurgery, Boston Children's Hospital, Harvard Medical School \\ ${ }^{4}$ Division of Epilepsy and Clinical Neurophysiology, Department of Neurology, Boston Children's Hospital, Harvard Medical School
}

Correspondence to: Christos Papadelis at christos.papadelis@childrens.harvard.edu

URL: https://www.jove.com/video/54883

DOI: doi:10.3791/54883

Keywords: Medicine, Issue 118, Pediatric Epilepsy, High Frequency Oscillations, Pre-surgical Biomarker, Magnetoencephalography (MEG), Electroencephalography (EEG), Source localization, Interictal Epileptiform Discharges (IEDs)

Date Published: 12/6/2016

Citation: Papadelis, C., Tamilia, E., Stufflebeam, S., Grant, P.E., Madsen, J.R., Pearl, P.L., Tanaka, N. Interictal High Frequency Oscillations Detected with Simultaneous Magnetoencephalography and Electroencephalography as Biomarker of Pediatric Epilepsy. J. Vis. Exp. (118), e54883, doi:10.3791/54883 (2016).

\section{Abstract}

Crucial to the success of epilepsy surgery is the availability of a robust biomarker that identifies the Epileptogenic Zone (EZ). High Frequency Oscillations (HFOs) have emerged as potential presurgical biomarkers for the identification of the EZ in addition to Interictal Epileptiform Discharges (IEDs) and ictal activity. Although they are promising to localize the EZ, they are not yet suited for the diagnosis or monitoring of epilepsy in clinical practice. Primary barriers remain: the lack of a formal and global definition for HFOs; the consequent heterogeneity of methodological approaches used for their study; and the practical difficulties to detect and localize them noninvasively from scalp recordings. Here, we present a methodology for the recording, detection, and localization of interictal HFOs from pediatric patients with refractory epilepsy. We report representative data of HFOs detected noninvasively from interictal scalp EEG and MEG from two children undergoing surgery.

The underlying generators of HFOs were localized by solving the inverse problem and their localization was compared to the Seizure Onset Zone (SOZ) as this was defined by the epileptologists. For both patients, Interictal Epileptogenic Discharges (IEDs) and HFOs were localized with source imaging at concordant locations. For one patient, intracranial EEG (iEEG) data were also available. For this patient, we found that the HFOs localization was concordant between noninvasive and invasive methods. The comparison of iEEG with the results from scalp recordings served to validate these findings. To our best knowledge, this is the first study that presents the source localization of scalp HFOs from simultaneous EEG and MEG recordings comparing the results with invasive recordings. These findings suggest that HFOs can be reliably detected and localized noninvasively with scalp EEG and MEG. We conclude that the noninvasive localization of interictal HFOs could significantly improve the presurgical evaluation for pediatric patients with epilepsy.

\section{Video Link}

The video component of this article can be found at https://www.jove.com/video/54883/

\section{Introduction}

Pediatric epilepsy is a common neurological disorder with a prevalence rate of 4 - 6 per 1,000 children ${ }^{1}$. It can have a major impact on children's development $^{2}$ and may significantly affect their adult life. Long-term follow-up studies in childhood-onset epilepsy indicate that approximately $30 \%$ of patients with epilepsy become medically intractable ${ }^{3-6}$, and usually require resective epileptic surgery. In many of these patients, epilepsy surgery leads to significant reduction in seizure frequency and often to seizure freedom. To be successful, epilepsy surgery should achieve a seizure-free state with minimal or no functional deficits. This requires careful delineation of the Epileptogenic Zone $(E Z)^{7}$, the 'area of cortex that is indispensable for the generation of epileptic seizures' ${ }^{8}$. The EZ cannot be measured directly; its location is estimated based on concordant data from a multitude of tests that identify other cortical zones. Invasive intracranial electroencephalography (iEEG) serves as the gold standard for the localization of the seizure onset zone (SOZ), the region where seizures are generated and originate on ictal recordings. However iEEG is costly, reliant on cooperation of the child, carries some risk for infection and bleeding ${ }^{9}$, and may induce additional neurological damage during the implantation ${ }^{10}$. Furthermore, the recordings may lead to erroneous conclusions since large areas of the brain are left unexplored. Thus, a robust presurgical biomarker that helps in the identification of the EZ is needed to the success of surgical epilepsy treatment.

Pathological HFOs $(80-500 \mathrm{~Hz})^{11,12}$ have emerged over the last decade as a biomarker for the identification of the epileptogenic tissue that may improve the presurgical diagnosis and surgical outcome of patients with epilepsy ${ }^{13}$. Reports using microelectrodes combined with depth EEG electrodes showed the presence of HFOs in patients with epilepsy. HFOs were also found using standard macroelectrodes during the ictal 
and interictal periods. Recent studies have shown that HFOs identify the SOZ with higher sensitivity and specificity compared to the irritative zone $^{14,15}$, the zone that generates the IEDs, and that the surgical removal of the HFO-generating tissue correlates with better outcomes than the removal of the SOZ or the irritative zone ${ }^{15}$. HFOs are commonly categorized as ripples $(80-250 \mathrm{~Hz})$ or fast ripples $(250-500 \mathrm{~Hz})$. Fast ripples have been more closely linked to pathological activity and to the localization of the $\mathrm{SOZ}^{16}$, but investigations of human intracranial recordings indicate that both ripples and fast ripples increase in epileptogenic regions ${ }^{17}$.

Despite these promising findings, HFOs are not yet suited for the diagnosis or monitoring of epilepsy in clinical practice. Primary barriers remain: (i) the lack of a formal and global definition for HFOs; (ii) the consequent heterogeneity of the methodological approaches used for their study; and (iii) the practical difficulties to detect and localize them noninvasively from scalp recordings. The latter stems from the fact that electrodes are far away from the source of the signal, the signal might be blurred by background noise and muscle activity, and the signal might be distorted by the scalp or the fontanels and sutures in the skull, especially in infant patients. Moreover, it is difficult to distinguish between normal and abnormal HFOs ${ }^{18,19}$ since both ripples and fast ripples are present even in normal human brain tissue ${ }^{20}$. Early studies reported HFOs in scalp EEG in only a small $(0.2-3.4 \%)$ portion of patients with epilepsy ${ }^{21-23}$. However, recent studies have shown that HFOs can be detected noninvasively with scalp EEG. Ictally, HFOs have been reported at the onset of epileptic spasms in children $\left(50-100 \mathrm{~Hz}{ }^{24}, 40-120\right.$ $\left.\mathrm{Hz}^{25}\right)$, as well as at the onset of tonic seizures in Lennox-Gastaut syndrome $(50-100 \mathrm{~Hz})^{26}$. Interictal HFOs $(70-200 \mathrm{~Hz})$ were first observed on scalp EEG in children with sleep-induced electrical status epilepticus ${ }^{27}$. Then, interictal HFOs $(80-200 \mathrm{~Hz})$ were identified in the scalp EEG of patients with focal epilepsy with higher rates inside the $\mathrm{SOZ}^{28}$. Interestingly, HFOs were more frequent in patients with high numbers of interictal epileptiform discharges (IEDs), and they were found to be more specific than IEDs for the $\mathrm{SOZ}^{29}$, highlighting the relation of $\mathrm{HFOs}$ with epileptogenicity.

MEG seems to present significant advantages compared to scalp EEG for the noninvasive detection and localization of HFOs: (i) high frequency activity in MEG is less susceptible than EEG to contamination from muscular activity ${ }^{30-31}$, (ii) MEG signals are not distorted by skull conductivity and less distorted than EEG by unfused regions of the cranial bone such as fontanel or suture, and (iii) MEG sensor arrays have higher density compared to EEG that always faces the problem of salt bridges between electrodes when the head is small, as with children. Evidence from phantom constructions that simulate HFOs generators suggested that HFOs can be detected and localized with high localization accuracy (2 $-3 \mathrm{~mm}$ ) with $\mathrm{MEG}^{32}$. Several recent studies reported HFOs in the MEG signals recorded from patients with epilepsy in the ripple frequency band $^{33-38}$. Time-frequency analysis has shown that MEG data contain high frequency components related to the EZ ${ }^{33-36}$. However, only a few studies have identified interictal HFOs as visible events standing out of the background signal in the time domain, as typically done with $\mathrm{iEEG}^{37-38}$. Van Klink et al. ${ }^{37}$ detected HFOs in the ripple band using virtual channels constructed with beamforming techniques based on spatial information obtained from IEDs. Von Ellenrieder et al. ${ }^{38}$ detected HFOs in MEG signals from the physical sensors independently of the IEDs and used the Maximum Entropy on the Mean (MEM) method to localize their sources and to investigate their correlation with the EZ. Rampp et al. (2010) also detected epileptic high gamma oscillations with MEG, which were spike-locked or spike-independent, and localized this activity with minimum-norm source analysis ${ }^{39}$. They found that characteristics of these fast oscillations (i.e., clear onset of full-band average and maximum amplitude of oscillations) were highly associated with the SOZ. HFOs were also detected with MEG during ictal activity in pediatric patients with epileptic spasms ${ }^{40}$. However, MEG presents some distinct limitations compared to scalp EEG: (i) it is insensitive to sources that have a radial orientation with respect to the center of the head, (ii) it does not allow long recordings that increase the possibility to detect and record ictal events, and (iii) its sensors cannot conform to the shape of head of each individual since the helmet and sensor array within the helmet are all fixed in shape. Thus, the ideal setup that maximizes the possibility to detect and localize the epileptogenic activity is by combining information from both scalp EEG and MEG.

In this study, we aim to illustrate the methodology we follow for the noninvasive detection of interictal HFOs by using simultaneous recordings of scalp EEG and MEG from pediatric patients with medically refractory epilepsy. We present the setup of the recordings and the pipeline of data analysis using a semi-automated method that we have developed for the detection of HFO events in simultaneous MEG and EEG data. Finally, we also present the localization of the underlying generators of scalp HFOs, obtained by solving the inverse problem, and compare it with the $\mathrm{SOZ}$ as this was defined by the epileptologists.

\section{Protocol}

Ethics Statement: Experimental procedures here have been approved by the Institutional Review Board (IRB) of Massachusetts General Hospital, Boston, MA, USA. The following section will describe the experimental protocol for the noninvasive detection and source localization of HFOs using scalp EEG and MEG. Patient preparation is minimal, and the examination is generally well tolerated. The entire session lasts about 2 - $3 \mathrm{~h}$ with the actual recordings lasting around $60 \mathrm{~min}$.

\section{Patient's Preparation}

1. Ensure that the child is comfortable with the environment. NOTE: Allow young children to explore the testing environment and to see the testing equipment. Screen the patient regarding safety and consent by using a screening form. Ask the patient (or his/her parents) whether he/she had a clinical seizure within the last $2 \mathrm{~h}$.

2. Remove all metallic/magnetic materials and provide hospital-issued clothing to the patient. Remove the shoes since they are frequently magnetic. Check if the subject is free of magnetic artifacts by measuring MEG signals for a few minutes. Use a degausser for reducing artifacts from implanted materials, such as dental works. NOTE: The demagnetizer should not be applied if there is any possibility that ferromagnetic objects are within the body.

3. Measure the maximum head circumference to select the appropriate EEG cap size for the child. Use a measuring tape and hold it to the nasion. Then measure around the head around the maximum circumference $(\sim 1 \mathrm{~cm}$ above the inion).

4. Place the EEG cap on the head according to the International $10-20$ system. Cleanse the skin where each electrode is located and apply paste/gel for each electrode. NOTE: More details regarding scalp EEG recordings in children are provided elsewhere ${ }^{41}$. 
1. Place the ground and reference electrodes on the head. Connect additional electrodes for measuring horizontal and vertical electrooculography (EOG), electrocardiography (ECG), electromyography (EMG) and additional EEG electrodes at locations covering the temporal regions (T1/T2).

NOTE: The EOG, ECG, and EMG aid the identification of eye movements, magnetocardiographic contamination, muscle activity, and also to monitor the patient's state.

5. Ensure that the EEG channels have good contact with the scalp by positioning the sensors individually. Gently twist each sensor from side to side to move hair out of the way. Measure all electrode impedances with an EEG ohmmeter in order to be below $10 \mathrm{KOhm}$.

6. Place four HPI coils on the head: two behind the earlobes and two on the forehead at approximate symmetrical locations. NOTE: The HPI coils help to localize the relative position of the patient's head with respect to the location of the MEG sensors in 3D space. The number of HPI coils may differ depending on the vendor of the MEG system.

7. Obtain the locations of the HPI coils and the EEG electrodes using a digitizer.

NOTE: The digitizer records the coordinates of a sensor in the 3D space. The sensor is placed on the tip of a stylus. The locations of the HPI coils must be known with respect to the head anatomy and the locations of the MEG sensors.

8. Obtain the locations of fiducial landmarks including left/right preauricular points and nasion using the digitizer. Digitize additional points (approximately 300 points) to obtain precise head shape.

9. Transfer the patient into the magnetic shielded room (MSR) ${ }^{42}$, where the MEG system is located. NOTE: The MSR is a shielded environment that minimizes the interference of MEG recordings from external electromagnetic sources (i.e., power lines, radiofrequency signals from portable devices, electrical devices and computers, magnetic fields from moving magnetized objects such as cars, elevators, and trains). It consists of three nested layers. Each layer is made of a pure aluminum layer plus a high permeability ferromagnetic layer (i.e., mu-metal, an alloy consisting mostly of nickel and iron).

10. Lay down the patient on the bed, put his/her head into the MEG helmet, and apply appropriate pads/sponges under the patient's head for comfort.

11. Connect the HPIs, the EEG leads, the EOG, the ECG, the EMG, and the additional electrodes to the recording machine. Adjust the patient's head position in the scanner ensuring that it is located as deep as possible in the helmet.

\section{Data Acquisition}

1. $M E G$ and EEG

NOTE: MEG/EEG data acquisition is performed based on the method described in a previous study ${ }^{42}$. More details about the clinical use of MEG in pediatric epilepsy can be found elsewhere ${ }^{43,44}$.

1. Record MEG signals with a whole-head MEG system.

NOTE: The MEG system employs thin-film sensors of two types (planar gradiometers and magnetometers) integrated on 102 sensor elements. Each element contains a magnetometer that consists of a single coil, and two orthogonal planar gradiometers that consist of a "figure-of-eight"-type coil configuration. The magnetometer measures the magnetic flux perpendicular to its surface and the gradiometers measure the difference between the two loops of the "eight", or the spatial gradient. The MEG system has 204 planar gradiometers and 102 magnetometers (306 sensors in total). MEG systems from different vendors have different number and types of coils (i.e., axial gradiometers).

2. Record simultaneously EEG signals using a nonmagnetic 70 -channel electrode cap with $\mathrm{Ag} / \mathrm{AgCl}$ sintered ring electrodes and additional electrodes in $\mathrm{T} 1 / \mathrm{T} 22^{42}$. Use a common reference montage.

3. Close the door of the MSR. Communicate with the patient via an intercom system to check if he/she feels comfortable. Ask the parent to stay inside the MSR during the recording if the child feels uncomfortable to stay alone.

4. Start the recordings by clicking the 'Go' button in the MEG acquisition software. Use a high sampling rate of $1 \mathrm{KHz}$ (or more). Use a low-pass Infinite Impulse Response (IIR) filter of $6^{\text {th }}$ order at $400 \mathrm{~Hz}$. Check online all the recorded signals. Fix bad MEG channels by using a sensor tuner.

NOTE: Bad MEG channels are defined sensors (gradiometers or magnetometers) that have a relatively high level of white noise (above 2 to $5 \mathrm{fT} / \sqrt{\mathrm{Hz}}$ for magnetometers) or sensors that record spurious environmental electromagnetic noise. This is usually happening when the sensors are exposed to strong (relative to the signals measured) magnetic fields and specific parts of the coils "trap" the magnetic flux destroying the superconductivity. A sensor tuner then is used that heat the coil by applying an electric current through it. This procedure is called tuning and is used when the white noise level of the sensor is above a specific threshold (i.e., 2 - 5 $\mathrm{fT} / \mathrm{VHz}$ ). Some MEG systems do not have sensor tuners.

5. Measure the patient's head position by clicking the 'Measure' button in the MEG acquisition software. If the patient's head is not wellcovered by the sensory array, ask the patient to move his/her head deeper into the helmet.

NOTE: This maneuver activates the $4 \mathrm{HPI}$ coils by applying transient oscillatory electrical signals through the coils that generate artificial magnetic fields. These fields are detected by the MEG sensors, thus the head position is determined. The procedure may differ between different MEG vendors.

6. Record MEG, EEG, and peripheral recordings by clicking the 'Record' button in the MEG acquisition software (i.e., ECG, EOG, and EMG) for $\sim 60 \mathrm{~min}$.

NOTE: The data is stored as a fif file in the Redundant Arrays of Independent Disks (RAID). The file type is different for other MEG vendors.

7. When the recording ends, open the MSR, disconnect the cables, and take out the patient from the MSR room. Remove all the tapes, electrodes, HPI coils, and EEG cap gently. Provide head washing for the patient.

8. After the acquisition is completed, record the magnetic signals of the empty MSR without the patient. Start the recordings by clicking the 'Go' button in the MEG acquisition software. Record MEG data for 2 min using the same parameters as in step 2.1.4. NOTE: This data is used for estimating the environmental electromagnetic noise.

2. MRI 
1. Acquire anatomical MRI data with magnetization-prepared rapid acquisition gradient-echo sequences (MPRAGE; TE $=1.74 \mathrm{~ms}$, TR $=2,520 \mathrm{~ms}$, voxel size $=1 \times 1 \times 1 \mathrm{~mm}$ ) with a high-resolution $3 \mathrm{~T}$ scanner. Details about the MRI scanning protocol can be found elsewhere ${ }^{45}$

NOTE: We do not perform the MRI scan the same day as the MEG session in order to avoid artifacts in the MEG recordings due to magnetization of possible patient's metallic implants, such as from dental works.

\section{Identification of Interictal Activity}

1. Open the data using Brainstorm ${ }^{46}$, which is documented and freely available for download online under the GNU general public license.

2. Select visually portions of the EEG data with interictal activity occurring at least $2 \mathrm{~h}$ apart from clinical seizures.

NOTE: Figure 1 presents a portion of EEG and MEG data with frequent IEDs.

1. Identify empirically well-defined IEDs in the EEG signals: this includes spikes $(20-70 \mathrm{~ms})$ and sharp waves $(70-200 \mathrm{~ms})^{47-48}$. NOTE: The clinical significance of both types of IEDs in epileptic focus localization is equivalent.

2. Try to identify (if possible) portions of the recordings with: (i) minimal motion artifacts, (ii) more than 3 - 4 IEDs per $10 \mathrm{~s}$ display, and (iii) slow-wave non-REM sleep that usually present a high number of $\mathrm{HFOs}^{49}$.
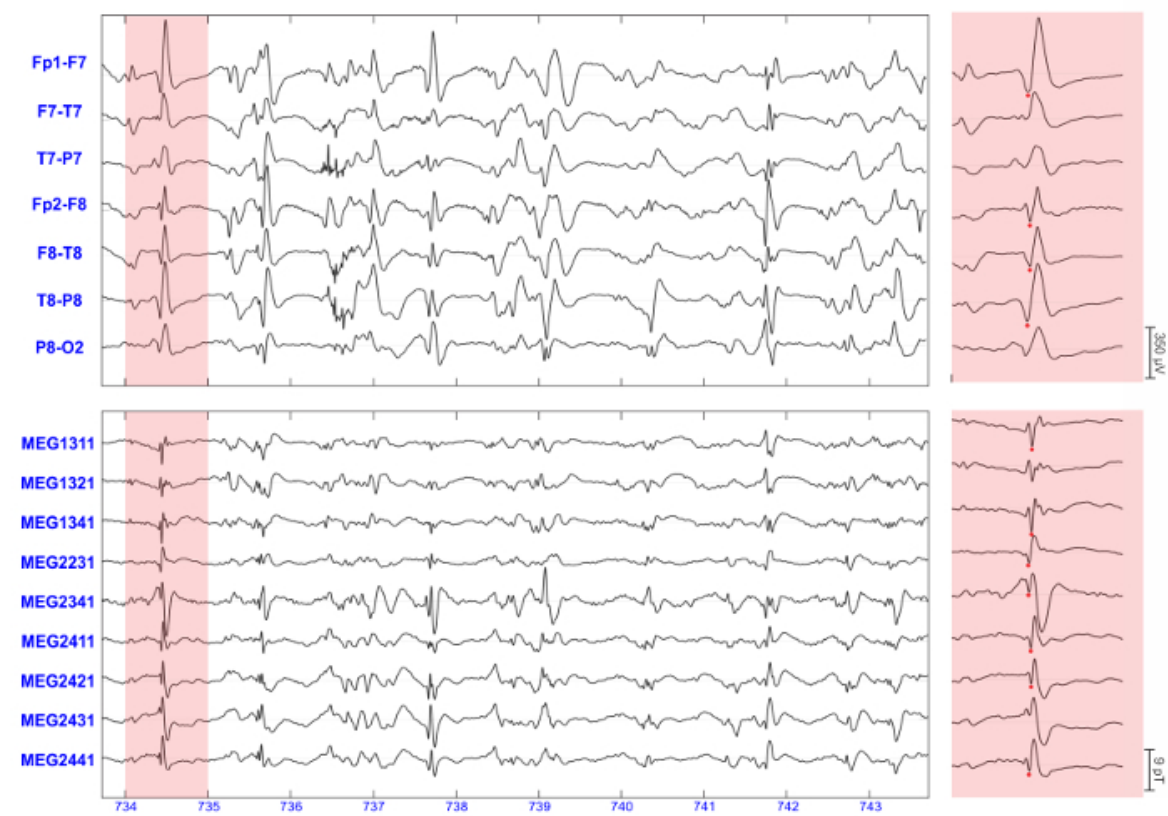

Figure 1: IEDs in EEG and MEG Signals. Portion of simultaneously recorded scalp EEG and MEG signals with frequent IEDs. $1 \mathrm{~s}$ of highlighted section that contains one sharp wave is presented on the right panels in an extended time scale display. Red dots indicate the peak of the IEDs.

Please click here to view a larger version of this figure.

3. Using Brainstorm, display the data with standard display settings (10 s/page). Go to the Filter tab and put the following filter display parameters: high-pass filter: $1 \mathrm{~Hz}$, Low-pass filter: $80 \mathrm{~Hz}$, Notch filter: 50 or $60 \mathrm{~Hz}$ (according to the frequency of the power line). Inspect the data and identify portions of data with IEDs.

NOTE: Only portions of signal with IEDs will be scanned to look for HFOs (step 3.4). The selected filters are for visualization only; they have not been applied to the data. In order to apply these filters permanently to the data, use a band-pass Butterworth filter ( $4^{\text {th }}$ order) following the instructions in Brainstorm website (http://neuroimage.usc.edu/brainstorm/).

4. Mark the peak of each IED occurring in both EEG and MEG data (see red spots in Figure 1).

NOTE: More details about marking IEDs using Brainstorm can be found elsewhere (http://neuroimage.usc.edu/brainstorm/Tutorials/Epilepsy).

\section{Semi-automated Detection of HFOs in Simultaneous Scalp EEG and MEG Data}

NOTE: Here we describe a semi-automated method to detect HFOs, which includes an automated detection (step 4.1; Figure 2), followed by a visual review of the automatically detected HFOs (step 4.3). In order to avoid the spurious oscillations of sharp transients as true ripples and to ensure that the HFOs are not due to a filtering phenomenon, we followed the latest suggestions in the relevant literature: we required the HFOs to have a minimum number of 4 oscillations since it has been observed that the impulse response of the filter has fewer oscillations than the chosen number of cycles ${ }^{50}$, we used the Finite Impulse Response (FIR) filter to minimize ringing effect and the "Gibbs" phenomenon ${ }^{50}$, we required the candidate HFO events to be inspected also visually by an expert to check whether the HFOs were also visible overlaid on the IEDs ${ }^{50,51}$, and we required an isolated island to be observed in the time-frequency plain because a sharp event and an oscillation have different signatures: a real $\mathrm{HFO}$ is represented by an isolated peak in the time-frequency plot (restricted in frequency, as "island") located in the band of 80 - $500 \mathrm{~Hz}$, while a transient event generates an elongated blob, extended in frequency ${ }^{50,52,53}$. 


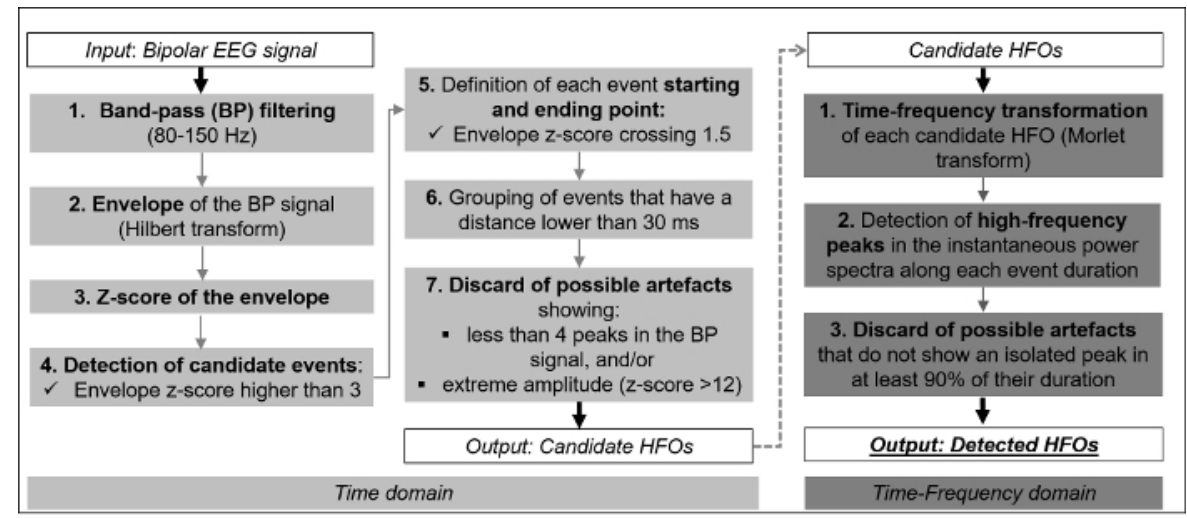

Figure 2: Schematic Diagram of the Algorithm Steps. The algorithm works in two stages: the first one identifies candidate HFOs from each EEG signal in the time domain (left and middle columns); the second one classifies the previously detected candidate events in order to distinguish real HFOs from artifacts in the time-frequency domain (right column). Please click here to view a larger version of this figure.

1. Automatic HFO detection

NOTE: Figure 2 describes the flow chart of the automatic detection of HFOs on each EEG signal. The goal of the developed method is to reduce the burden for the EEG expert of marking HFO events on each EEG channel using a $2 \mathrm{~s} /$ page display that is recommended for the visual inspection of HFOs. An HFO was defined as an event within the ripple frequency band $(80-250 \mathrm{~Hz})$, which has at least 4 oscillations of sinusoidal like morphology standing out from the surrounding background ${ }^{54}$ (step 4.1.1), and which appears as a short-lived event with an isolated spectral peak at a distinct high frequency ${ }^{14}$ (step 4.1.2).

1. Detection of candidate HFOs in the time domain

1. Band-pass (BP) filter the EEG signals between 80 and $250 \mathrm{~Hz}$, restricting the frequency content of the signals to the ripple band of interest. NOTE: It is recommended to use a FIR filter to minimize ringing effect and the "Gibbs" phenomenon ${ }^{55}$, and zero-phase digital filtering to avoid phase distortion.

2. Calculate the envelope of the BP signal using the Hilbert transformation. Calculate the mean and the standard deviation (SD) of the envelope over $10 \mathrm{~s}$ sliding windows centered on each point of the time-series. Estimate the overall mean and SD using the median value over all the windows (in order to obtain values that are robust to the possible presence of portions of the signal with many HFOs and high SD).

3. Calculate the $z$-score of the envelope and mark a candidate HFO every time the z-score is higher than the minimum threshold, set equal to $3^{56}$.

4. Define the starting and ending points of the detected event as the upward and downward crossings of half the threshold. Consider the HFOs with an inter-event interval of less than $30 \mathrm{~ms}$ as one single HFO. Calculate the number of peaks in the BP signal between the HFO starting and ending points, and discard events with less than 4 peaks. Also, discard events with a zscore higher than 12.

NOTE: Modify your maximum z-score threshold according to the amplitude of the artifacts that may occur in your recordings. Events with a low number of oscillations can be caused by filtering effects ${ }^{57,58}$, whereas events with extremely high amplitude can be due to muscle or electrode artifacts.

2. Reject possible artifacts in the time-frequency domain.

NOTE: This step is necessary to distinguish real HFOs from events that might be elicited by other EEG activity and filtering artifacts, whose frequency content is not restricted to the frequency band of interest. It is based on the assumption that a real HFO appears as a short-lived event with an isolated spectral peak at a distinct frequency above $80 \mathrm{~Hz}$, in contrast with a transient event that generates an elongated blob extended in frequency ${ }^{59}$. Figure 3 illustrates an example of a detected HFO showing the BP filtered EEG signal (upper panel), its envelope (middle panel), and the corresponding time-frequency plane (lower panel), during the period of [-0.5, +0.5] $S$ around the HFO peak. The display of the time-frequency plane is restricted from $80-150 \mathrm{~Hz}$ because no prominent activity was observed for frequencies above $150 \mathrm{~Hz}$.

1. Transform all candidate HFOs events into the time-frequency space using the Morlet transformation in the frequency range from $1 \mathrm{~Hz}$ to the highest frequency of interest, i.e., $250 \mathrm{~Hz}$ (central frequency $=1 \mathrm{~Hz}$, Full-Width-At-Half-Maximum $=3 \mathrm{~s}$ ).

2. Analyze the instantaneous power spectra of the time-frequency representation over each time point of the event duration. For each power spectrum, follow the automatic criteria described by Burnos et al. ${ }^{56}$ to detect the peak in the high-frequency band and to verify whether it is clearly distinct from the closest peak in the lower frequency range. Discard HFOs that do not show a power spectrum with an isolated high-frequency peak in at least $90 \%$ of the time points.

2. Sort all the detected HFOs events by their temporal occurrence across channels. Group together all the consecutive HFOs whose duration overlaps. Keep only groups of HFOs involving at least two EEG channels for further analysis.

NOTE: The algorithm requests the HFOs to occur in at least 2 channels in order to avoid capturing spurious random artifacts, which may resemble real HFOs and occur in single EEG leads. Two consecutive HFOs are considered as overlapping when the starting time of the second HFO precedes the starting time of the first one. 


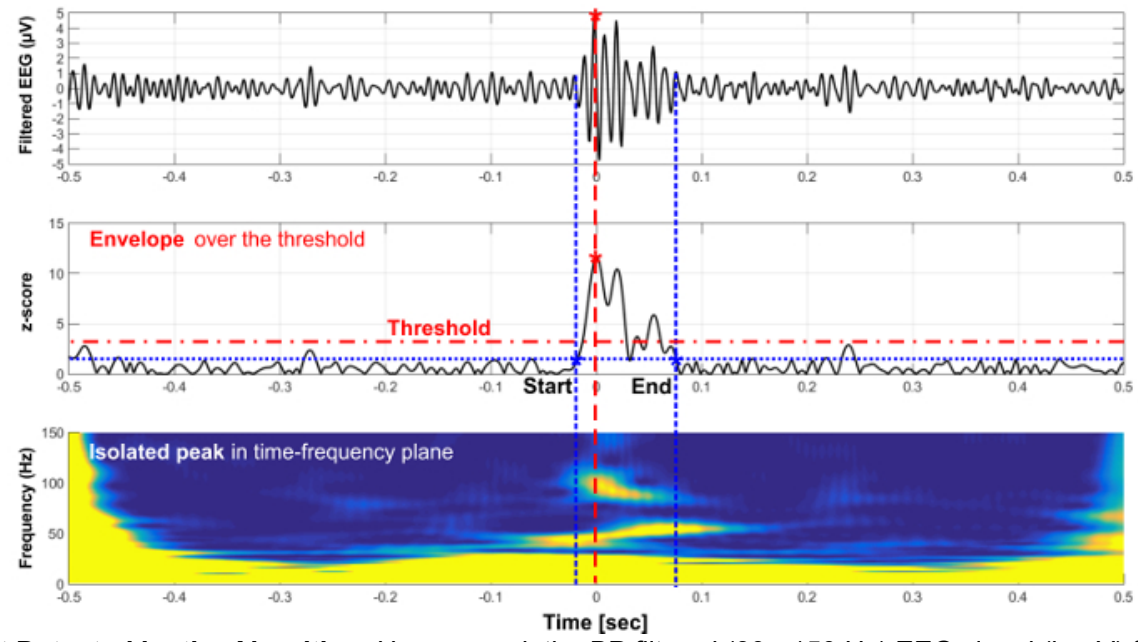

Figure 3: HFO Event Detected by the Algorithm. Upper panel: the BP filtered ( $80-150 \mathrm{~Hz})$ EEG signal (in $\mu \mathrm{V})$ from one channel (F8 T8) from patient 1. Middle panel: the envelope of the BP signal (z-score). The peak of the envelope (red asterisk) indicates the timing of the HFO peak (red vertical dotted line). The blue asterisks mark the upward and downward crossings of half the threshold (blue dotted line), which indicate the start and end time-points of the HFO (blue vertical dotted lines). Lower panel: the time-frequency analysis plane. Note the isolated peak in the ripple frequency band $(\sim 100 \mathrm{~Hz})$ around the peak of the HFO. Please click here to view a larger version of this figure.

3. Visual review of HFO events

NOTE: Part of the visual review is based on the guidelines proposed by Andrade-Valenca et al. ${ }^{28}$ and Zelmann et al. ${ }^{60}$.

1. Vertically align 2 computer screens; one for the inspection of EEG and one for the inspection of MEG signals. Display the detected events on both the expanded ( 2 s/page) and typical scale (10 s/page) showing, respectively, the $80-250 \mathrm{~Hz}$ and the $1-40 \mathrm{~Hz} \mathrm{BP}$ filtered signals.

2. Ignore events cooccurring with muscle or electrode artifacts in the unfiltered EEG and MEG, as well as events with large frequency variability, irregular morphology, or large amplitude variations.

3. Observe the EOG and EMG signals during the detection of the HFOs and discard any event that is thought to correspond to EOG or muscular activity. Consider only the HFOs that overlap with EEG/MEG IEDs (detected in step 3.3) as they are more likely to be true HFOs ${ }^{15,28,56}$

NOTE: This approach offers high specificity at the cost of low sensitivity; thus, it provides confidence that the identified HFOs are of cortical origin.

4. Keep only HFO events that occur in both EEG and MEG signals at the same time.

\section{Source Localization of IEDs and HFOs}

1. Localize the generators at the peak of the MEG IEDs, marked in step 3.3, using the Equivalent Current Dipoles (ECD). Use the Minimum Norm Estimates software that is freely available (http://martinos.org/mne/stable/index.html). Consider only spikes with goodness-of-fit (GOF) $>80 \%$ and dipole moment $\mathrm{Q}<500 \mathrm{nA}-\mathrm{m}$. Overlay the ECD location on the MRI of each patient.

NOTE: The Maximum Entropy on the Mean (MEM) is an attractive alternative method that determines the location and extent of the sources $^{61}$

2. HFO source localization at both EEG and MEG using the wavelet Maximum Entropy on the Mean (wMEM) method (as proposed by von Ellenrieder et $a l^{38}$ ).

NOTE: The MEM is an efficient technique that has been successfully used to determine the location and extent of sources of epileptic activity $^{62-64}$. The WMEM is an extension of MEM that has been developed for localizing oscillatory activity as evaluated with realistic simulations $^{65}$. It decomposes the signal in a discrete wavelet basis before performing MEM source localization on each time-frequency box. Thus, wMEM is particularly well suited to localize HFOs.

1. Segment the MRI and obtain the cortical surface using Freesurfer ${ }^{66-67}$.

2. Solve the EEG/MEG forward problem with the boundary element method (BEM) for a 3-layer model using OpenMEEG ${ }^{68}$.

3. Resample the signals to $640 \mathrm{~Hz}$ in order to ensure that the second scale of the discrete wavelet transform corresponds to the frequency band of interest.

4. Estimate the noise covariance matrix in the data space independently for each HFO, based on the background in the ripple band in a $150 \mathrm{~ms}$ window immediately before each HFO. Perform the source localization for each HFO in the ripple band and average along the HFO duration. Note: The resulting map consists of a cortical activation value associated to each vertex of the cortical tessellation.

5. Normalize each map in order to have a maximum activation value equal to 1 for each HFO.

6. Compute the average of the activation values across all HFOs at each vertex. Apply a threshold of $60 \%$ of the maximum activation in order to display the final maps over the cortical surface.

\section{Validation}

1. Intracranial EEG (iEEG): 
1. Acquire extra-operative iEEG by employing subdural grids and/or stereotactically guided depth electrodes. Guide the placement of the electrodes based on the results of previous presurgical evaluation tests and the clinical hypotheses to be addressed, which is specific for each patient.

NOTE: Intracranial EEG is recorded with a digital EEG system using a $2 \mathrm{KHz}$ sampling rate, as part of the presurgical evaluation. Subdural grids are typically the best choice if the topography of the cortical seizure area is important to resolve, and if mapping of the eloquent cortex is critical (e.g., with an epileptogenic area near primary motor cortex, where the exact boundaries can be estimated by stimulation mapping and ictal intracranial EEG analysis).

2. Definition of the SOZ:

1. Identify the $\mathrm{SOZ}$ as defined by an expert epileptologist based on the clinical information available for each patient.

NOTE: The SOZ is defined as the area showing the earliest and rapid spread of iEEG change (ictal discharge) from baseline prior to or concomitant with the clinical onset. All channels involved at the beginning of the ictal electrographic discharge, usually the first $5 \mathrm{~s}$, were considered as the SOZ. In patients where the seizures are originating from more than one area independently of each other, all contacts within the different SOZs are regarded as SOZ contacts. The expert is blind to the HFO source localization results at the time of defining the SOZ. The clinical information also includes: ictal and interictal iEEG findings, visible lesion on the MRI, ictal and interictal scalp EEG findings.

3. Identification of the HFO channels:

1. Detect the HFOs in each intracranial electrode as described in 4.1. Use Kittler's method to determine the threshold based on the histogram of the number of HFOs from all channels, then apply bootstrapping and calculate the mean as the final threshold ${ }^{69}$. Finally, identify the channels with an HFO rate above the threshold.

4. Compare the HFO localization with the SOZ identified in 6.2 as the gold standard for the source localization results.

\section{Representative Results}

Pediatric patients with refractory epilepsy were recruited from the Epilepsy Clinic of Boston Children's Hospital (Boston, USA). Here, representative data from 2 patients are presented: a 15-year-old girl with encephalomalacia of the right middle cerebral artery (MCA) region (patient 1), and an 11-year-old boy with left parietal/superior temporal encephalomalacia (patient 2). The data were collected as part of their presurgical workup for surgery. Simultaneous MEG and EEG recordings were performed at the Athinoula A. Martinos Center for Biomedical Imaging.

For both patients, HFOs were identified in the ripple frequency band $(80-150 \mathrm{~Hz})$ occurring in both EEG and MEG and overlying IEDs. Figure 4 presents $10 \mathrm{~s}$ of simultaneous scalp EEG and MEG data with interictal activity (upper panels) from patient 1 . The same figure also reports an extended time scale portion ( $2 \mathrm{~s}$ ) of the signals, which shows the detected HFOs in the time domain (middle panels) and in the time-frequency plane (lower panel). This patient showed a rate of $8.8 \mathrm{HFOs} / \mathrm{min}$. For clarity, the display of the time-frequency plane is restricted from $80-150$ $\mathrm{Hz}$ because no prominent activity was observed for frequencies above $150 \mathrm{~Hz}$. In patient 1, the automatic detection identified $248 \mathrm{HFOs}$ events within $8.65 \mathrm{~min}$ of recordings. After visual review of the detected events, 76 events were kept that were considered to be real HFOs, obtaining a rate of $8.8 \mathrm{HFOs} / \mathrm{min}$. 

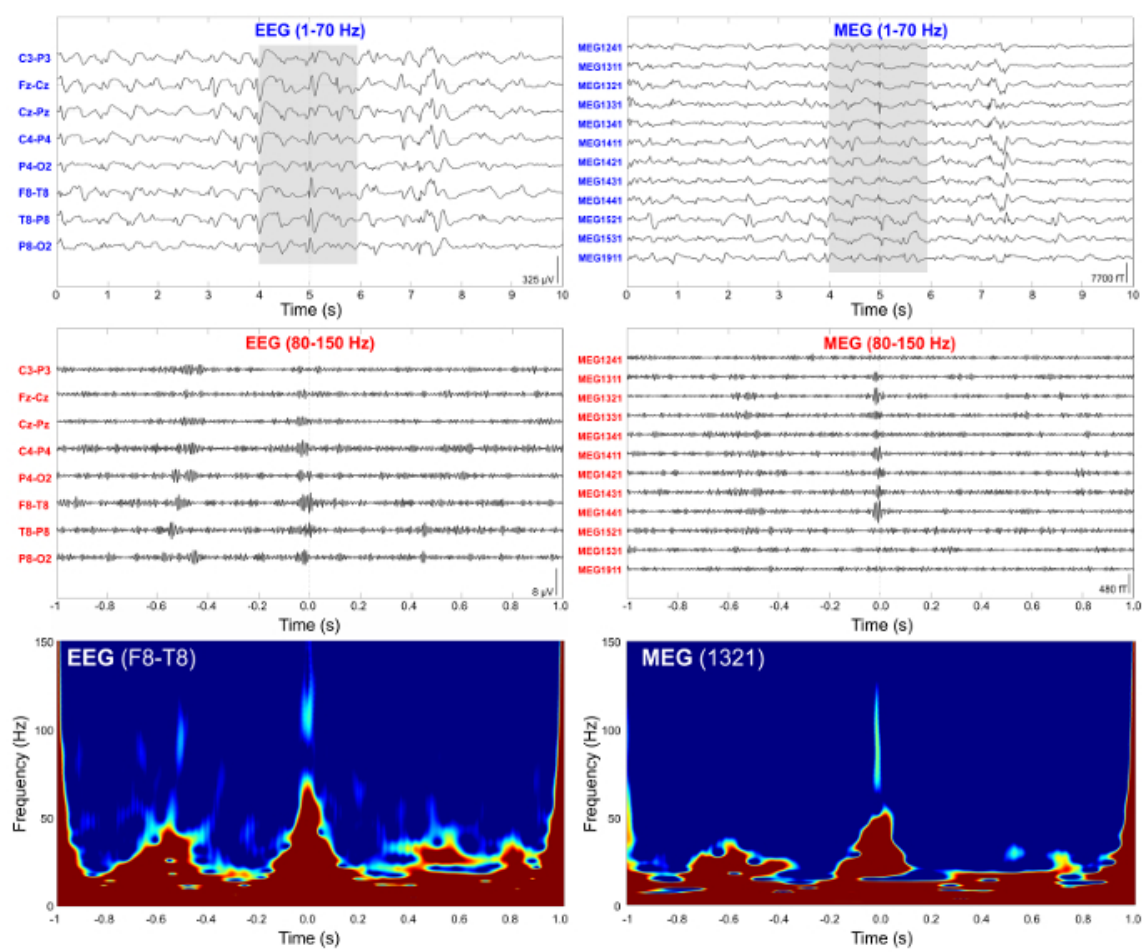

Figure 4: HFOs Overlaid on IEDs Detected from Scalp EEG and MEG. Upper panel: IEDs from patient 1 detected from scalp EEG (left) and MEG (right). $10 \mathrm{~s}$ of data filtered from $1-70 \mathrm{~Hz}$. Middle panel: Extended time scale display $(2 \mathrm{~s})$ of the EEG and MEG data highlighted with grey background in the upper panel. Data are filtered from $80-150 \mathrm{~Hz}$. Lower panel: Time-frequency plane of 2 representative EEG and MEG channels. Please click here to view a larger version of this figure.

Figure 5 presents the HFOs localization results for patient 1 from both scalp EEG and MEG. Both techniques localized the HFOs at the vicinity of the lesion in a location close to the right temporo-parietal junction. MEG and EEG presented slightly different localization results: MEG activity was located more anteriorly compared to EEG and closer to the posterior boundary of the lesion. The distance between the two maxima of wMEM activity for the MEG and EEG was $15.0 \mathrm{~mm}$. HFOs localization was also adjacent to the irritative zone as this was defined by MEG. The Euclidian distance between the averaged ECDs and the maximum wMEM activity was $18.7 \mathrm{~mm}$ for the MEG and $28.0 \mathrm{~mm}$ for the scalp EEG. For patient 2, we identified 8 min of data during slow-wave non-REM sleep with IED and minimal artifacts that we used for the HFO analysis.

The number of scalp HFOs was significantly lower than in patient 1 showing a rate of $0.4 \mathrm{HFOs} / \mathrm{min}$. Figure 6 presents the source localization results of scalp HFOs for MEG and scalp EEG, the IEDs localized by MEG using ECDs, and the location of HFOs detected by iEEG. The distance between the 2 maxima of wMEM activity for the MEG and EEG was $16.4 \mathrm{~mm}$. The distance between the averaged ECDs and the maximum wMEM activity was $10.9 \mathrm{~mm}$ for the MEG and $24.1 \mathrm{~mm}$ for the EEG. The HFO zone identified noninvasively by scalp EEG and MEG was at the same location where the HFOs were identified invasively with iEEG. The electrodes with the highest number of HFOs were LA51, LA52, and LA53, as shown in Figure 6, whose localization was concordant with the HFO activity localized noninvasively. 


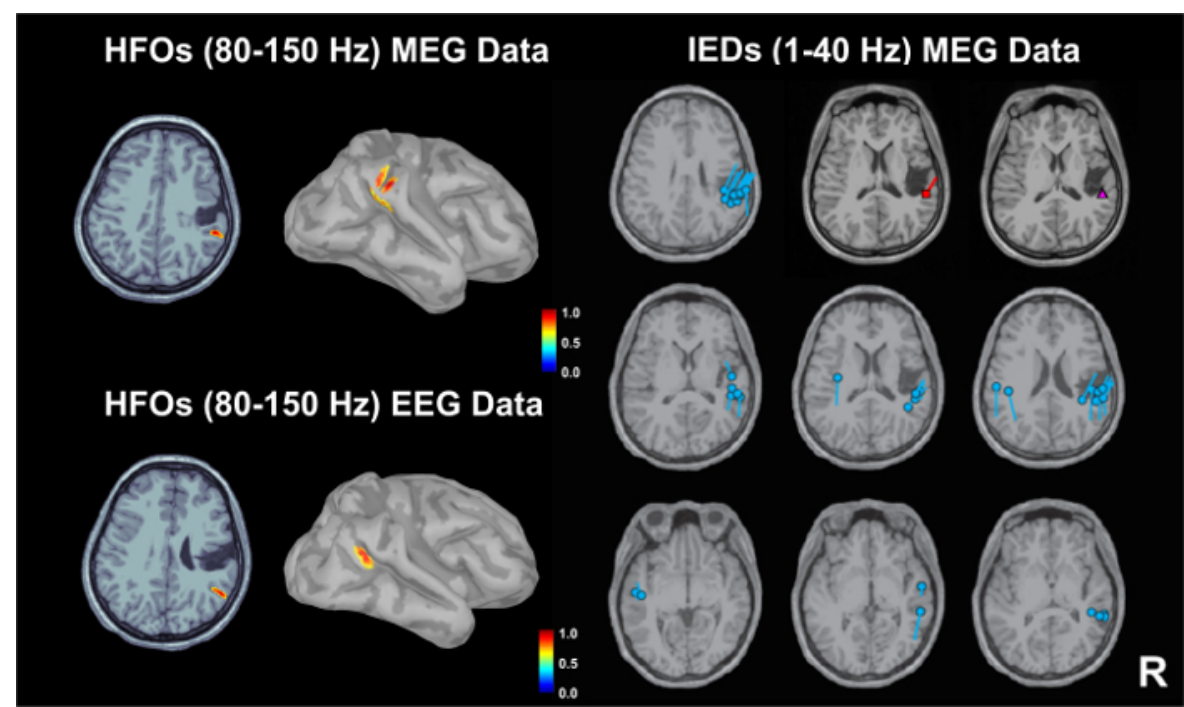

Figure 5: Localization of HFOs and IEDs. HFOs zone localized by using the wMEM with MEG and EEG from patient 1 overlaid on patient's MRI (left). The HFOs map represents the average of the normalized activation values thresholded at $60 \%$ of the maximum activity. IEDs localized by ECDs with MEG (right). The locations and orientations of the ECDs are indicated in cyan. The purple triangle indicates the location of the averaged ECDs (46 ECDs), and the red rectangle the location of the ECD from averaged IEDs (28 IEDs).

For the averaging of the IEDs, we identified spikes with similar morphology. Please click here to view a larger version of this figure.
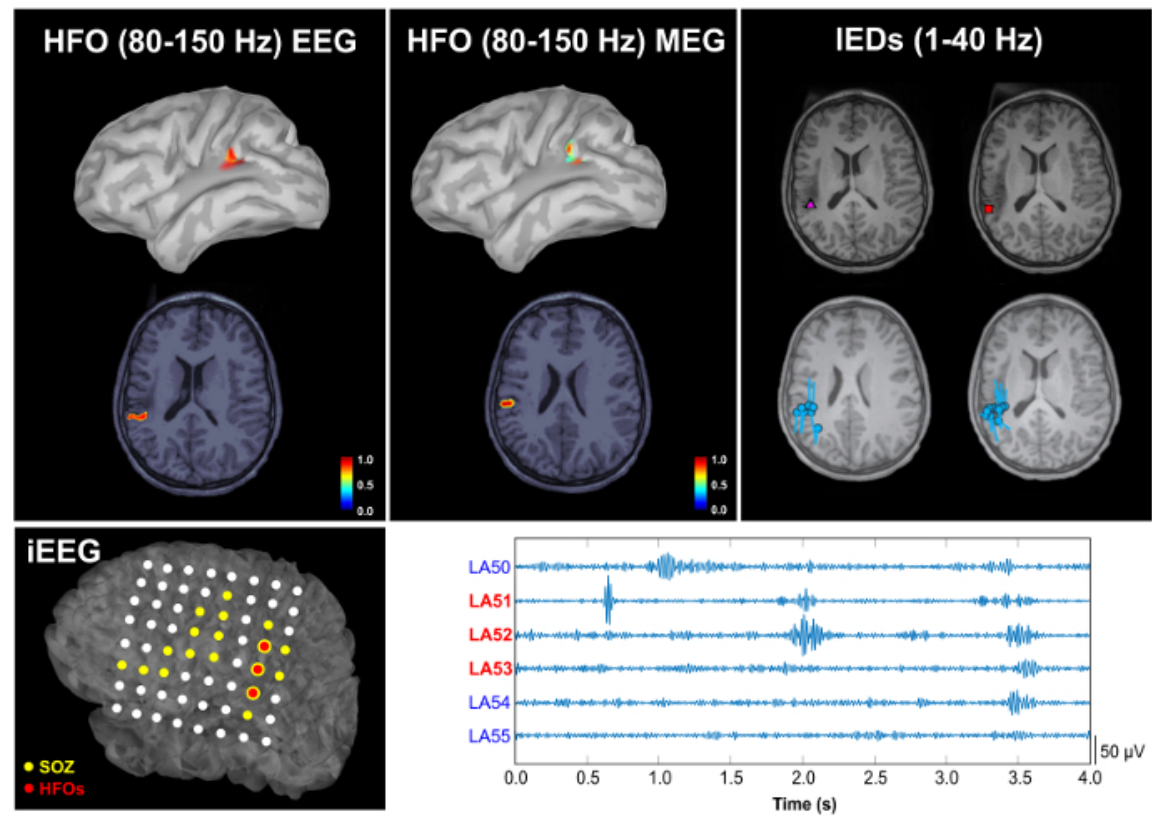

Figure 6: Spatial Concordance between the HFO Zone Localized Noninvasively with Scalp EEG and MEG, the Irritative Zone, and the HFO Zone Localized Invasively with iEEG. Upper panels: Localization of HFOs from patient 2 with scalp EEG (left) and MEG (middle), and localization of IEDs with MEG (right) overlaid on patient's MRI. The HFOs map represents the average of the normalized activation values thresholded at $60 \%$ of the maximum activity. Cyan circles and bars indicate the locations and orientations of the ECDs. The purple triangle indicates the location of the averaged ECDs (30 ECDs), and the red rectangle the location of the ECD from averaged IEDs (21 IEDs). Lower panels: Localization of HFOs on iEEG (left) and $2 \mathrm{~s}$ of recordings from channels with the highest HFO rate. Implanted electrodes are shown on the patient's MRI. The electrode location is obtained by co-registering the post-implanted computed tomography (CT) and MR images. The electrodes with the highest rate of HFOs are highlighted on the map of the grid implanted on the patient's cortex. Please click here to view a larger version of this figure.

The representative results presented here indicate the potential use of the described technique in the clinical setting for the noninvasive identification and localization of HFOs during the pre-surgical evaluation of pediatric patients with medically refractory epilepsy. For both patients, HFOs were localized concordant for scalp EEG and MEG (see Figure 5). Localization differences of few mm may be attributed to the superior localization ability of high-density MEG compared to scalp $E E G^{70}$, or may represent different underlying generators ${ }^{71}$. The localization was also concordant with the irritative zone as this was defined by MEG (see Figure $\mathbf{5}$ - right panel). In patient 2, data from iEEG were also available. The HFOs zone localized noninvasively with scalp EEG and MEG was concordant with the HFOs zone defined invasively with iEEG (see Figure 6). The outcomes of the proposed method can guide the placement of grids during epilepsy surgery for the potential identification of the EZ. 
Discussion

Converging evidence from animal and human studies has shown that HFOs are a new potential biomarker for epileptogenic tissue. Despite this evidence, HFOs have very limited use in clinical practice for the diagnosis or monitoring of epilepsy, mostly because: (i) there is no formal and global definition for HFOs; (ii) different research groups use different methodology for recording and analyzing the data; (iii) the noninvasive detection of HFOs with neuroimaging techniques is challenging; and (iv) the review process of HFOs is time-consuming and impractical, especially for multichannel EEG or MEG recordings with a high number of sensors. In an effort to provide a global standardized methodology that promotes the systematic use of HFOs in clinical practice, the methodology that is followed at Boston Children's Hospital for the noninvasive recording, detection, and localization of interictal HFOs from pediatric patients with epilepsy is presented. Representative results of HFOs detected with simultaneous scalp EEG and MEG from two children with medically refractory epilepsy are also presented.

\section{Critical steps within the protocol}

The proposed methodology includes the following critical steps: (i) the performance of high Signal-to-Noise-Ratio (SNR) EEG and MEG simultaneous recordings of interictal activity from pediatric patients with medically refractory epilepsy (steps 2.1.1 and 2.1.2); (ii) the careful preprocessing and selection of data with interictal discharges (steps 3.1 and 3.2); (iii) the visual review of the identified HFOs events with high specificity (steps 4.3.1, 4.3.2, and 4.3.3); and (iv) the reliable localization of the HFOs using an appropriate localization method (step 5.2).

The most critical step in this protocol is the visual review of the HFO events identified by the automatic detector. A rigorous review of the automatically detected HFOs is crucial to discard HFOs of noncerebral origin. However, fatigue or distraction of the human reviewer during the visual inspection of multichannel EEG and MEG data may lead to errors, reducing the specificity of the detection process.

\section{Modifications and troubleshooting}

We avoid the use of the Signal Space Projection (SSP) and Signal Space Separation (SSS) methods ${ }^{72,73}$ in order to ensure that there was no distortion of the HFO activity from their application. These methods are often used by most of the users of the particular MEG vendor to suppress external interferences and to correct for head movements ${ }^{72}$. Further studies are necessary in order to ensure that the application of these methods do not affect or distort the HFO activity or do not produce spurious effects that may resemble human HFOs. Minor modifications of the minimum threshold of the z-score of the signal envelope (step 4.1.1.3) and the threshold of activation values (step 5.2.6) may be needed to improve the sensitivity of the algorithm in the detection of HFOs and restrict the localization of the HFOs zone in a more focal area.

\section{Limitations of the technique}

The described method presents limitations that should be further addressed in future studies. First, it does not consider HFOs occurring only in the MEG or EEG signals, and it does not include the automatic detection of HFOs in the MEG signals, which implies that some actual low SNR MEG HFOs might escape visual inspection ${ }^{74}$. Furthermore, the sensitivity and specificity of the proposed method to detect the HFOs and its ability to localize them with high accuracy should be validated with simultaneous recordings of scalp EEG, MEG, and iEEG ${ }^{75}$. Our data have shown that single ECDs indicated an extended irritative zone compared to the focal HFOs zone. However, when the ECDs were averaged, then the dipole location was quite close to the HFO zone for both patients. Our data are indicative of the specificity of the 2 methods showing a possible higher specificity of the HFO zone for epileptogenicity (particularly for patient 2 for whom the HFO zone was overlapping with the SOZ) compared to the irritative zone, although secure conclusions cannot be drawn from such a small cohort of patients. More importantly, the localization of the HFO sources does not directly imply localizing the EZ that is responsible for seizures. Our findings should be validated against the outcome of the epilepsy surgery that we plan to do in a future study. Finally, to record the EEG data, a 70-channel system was used. Yet, in most centers the standard clinical EEG setting is used that records data from 19 electrodes placed according to the $10-20$ system. More advanced pediatric EEG systems with much higher number of channels (up to 256) are currently available in the market. The use of these systems may further improve the localization accuracy of the HFOs zone detected with scalp EEG.

\section{Significance of the technique with respect to existing/alternative methods}

To our best knowledge, this is the first study that reports the noninvasive localization of interictal HFOs with simultaneous EEG and MEG, and also investigates the concordance of the localization results with those from intracranial recordings. The noninvasive recording, detection, and localization of HFOs is challenging. This is because HFOs are very weak signals generated by small brain regions on the order of cubic millimeters ${ }^{16,76}$ and furthermore hindered by noise and brain background activity. A recent study proposed that HFOs recorded non-invasively with scalp EEG represent the sum of activity of multiple spatially distributed focal and coherent sources ${ }^{60}$. So far, few studies ${ }^{28,29,37,38,60}$ managed to show that HFOs can be detected non-invasively using scalp EEG and MEG; even fewer localized this activity by solving the inverse problem $^{37-38}$.

Here, evidence of interictal HFOs are presented which have been detected with simultaneous scalp EEG and MEG from two pediatric patients with epilepsy. HFOs were localized by using a previously described framework ${ }^{38}$. The representative data suggest that the noninvasive localization of interictal HFOs is feasible by using source imaging techniques performed on either scalp EEG or MEG recordings, assuming that an appropriate localization technique is used. This is in line with a previous study that used a phantom construction resembling HFOs generators, which indicates that HFOs can be noninvasively detected and accurately localized with $\mathrm{MEG}^{32}$.

The detection and labeling of interictal HFOs is traditionally performed through the visual inspection of data from human EEG experts. Although this approach is often regarded as the gold standard, it presents serious limitations since it has poor inter-reviewer reliability ${ }^{77,78}$, and is not applicable to large MEG and EEG datasets with high number of sensors. Crucial to the application of HFOs in clinical practice is the development of algorithms that detect the HFOs automatically from scalp recordings reducing the need for human input. The visual identification of scalp HFOs is in fact quite challenging due to: (i) the low SNR of HFOs on the scalp; (ii) the lower rates of HFOs in scalp recordings compared to intracranial ones, which implies the analysis of much longer recording times; and (iii) the high number of channels to analyze, particularly in high-density EEG or MEG. Several algorithms for automatic and semi-automatic detection of HFOs have been proposed in the last decade ${ }^{54}$. 
Earlier detectors relied on thresholds in the time domain, in order to identify events that can be distinguished from ongoing background activity ${ }^{49,80}$. Recent advances suggest also incorporating information from the frequency domain, assuming that an HFO must appear as a shortlived event with an isolated spectral peak at a distinct frequency ${ }^{50,56,81}$. Semi-automated methods seem to be the most appropriate approach for the application of HFOs in clinical practice. These methods involve 2 stages: (i) initial automatic detection of events that has high sensitivity, and (ii) visual review of events by an expert, which has high specificity. This approach provides higher specificity compared to the fully automated methods and ensures that the final reviewed events are actual HFOs of cerebral origin.

Here, a semi-automated method is presented that enables the detection of HFOs from interictal scalp EEG and MEG recordings. The proposed method extends previously described techniques for the detection of HFOs from scalp EEG ${ }^{60}$ by incorporating in the identification criteria two important features: (i) the automatic time-frequency analysis of the HFO events; and (ii) the temporal concurrence of HFOs events in both MEG and EEG recordings.

\section{Future applications or directions after mastering this technique}

The reliable localization of HFOs with non-invasive neuroimaging methods, such as scalp EEG and MEG, is critical. Mastering, improving, and validating the proposed protocol will provide physicians with a reliable, noninvasively recordable biomarker for the identification of the EZ. The development of such a biomarker has the potential to reduce the requirement for long-term monitoring and invasive intracranial recordings leading to a significant improvement in the presurgical evaluation procedure in pediatric patients. It would not only help to identify the epileptogenic tissue for surgery, but would also permit definitive differential diagnosis of epilepsy from acute symptomatic seizures, requiring an entirely different treatment approach, and from non-epileptic seizures sparing the need for long-term monitoring in some patients. Further, this might allow assessment of efficacy of therapeutic interventions without waiting for another seizure to occur.

\section{Disclosures}

The authors have nothing to disclose.

\section{Acknowledgements}

This work is supported by the Research Grants Program of the Epilepsy Foundation \& American Epilepsy Society and the Faculty Career Development Fellowship of Harvard Medical School, Office for Faculty Development.

\section{References}

1. Cowan, L.D., Bodensteiner, J.B., Leviton, A., \& Doherty, L. Prevalence of the Epilepsies in Children and Adolescents. Epilepsia. 30 (1), 94-106 (1989).

2. Stores, G. School-children with Epilepsy at Risk for Learning and Behaviour Problems. Dev. Med. Child Neurol. 20 (4), $502-508$ (1978).

3. Sillanpää, M., Schmidt, D. Prognosis of seizure recurrence after stopping antiepileptic drugs in seizure-free patients: A long-term populationbased study of childhood-onset epilepsy. Epilepsy Behav. 8 (4), 713-9. (2006).

4. Geerts, A. et al. Course and outcome of childhood epilepsy: a 15-year follow-up. Dutch study of epilepsy in childhood. Epilepsia. $\mathbf{5 1}$ (7), 1189-1197 (2010).

5. Engel, J. Etiology as a risk factor for medically refractory epilepsy: A case for early surgical intervention. Neurology. 51 (5), $1243-1244$ (1998).

6. Kwan, P., \& Brodie, M. Early Identification of Refractory Epilepsy. N. Eng. J. Med. 342 (5), 314-319 (2000).

7. Datta, A., \& Loddenkemper, T. The Epileptogenic Zone. In: Wyllie, E. Wyllie's treatment of epilepsy: Principles \& Practice 5th Ed. Wolters Kluwer/Lippincott Williams \& Wilkins: Philadelphia, 818-827 (2011).

8. Rosenow, F. Presurgical evaluation of epilepsy. Brain. 124 (9), 1683-1700 (2001).

9. Önal, Ç., \& Otsubo, H. et al. Complications of invasive subdural grid monitoring in children with epilepsy. J. Neurosurg. 98 (5), $1017-1026$ (2003).

10. Jacobs, J., \& Zijlmans, M. et al. High-frequency electroencephalographic oscillations correlate with outcome of epilepsy surgery. Ann. Neurol. 67 (2), 209-220 (2010).

11. Bragin, A., Engel, J., Wilson, C., Fried, I., \& Buzsáki, G. High-frequency oscillations in human brain. Hippocampus. 9 (2), $137-142$ (1999).

12. Bragin, A., Engel, J., Wilson, C., Fried, I., \& Mathern, G. Hippocampal and Entorhinal Cortex High-Frequency Oscillations (100-500 Hz) in Human Epileptic Brain and in Kainic Acid-Treated Rats with Chronic Seizures. Epilepsia. 40 (2), 127-137 (1999).

13. Zijlmans, M., Jiruska, P., Zelmann, R., Leijten, F., Jefferys, J., \& Gotman, J. High-frequency oscillations as a new biomarker in epilepsy. Ann. Neurol. 71 (2), 169-178 (2012).

14. Crepon, B., \& Navarro, V. et al. Mapping interictal oscillations greater than $200 \mathrm{~Hz}$ recorded with intracranial macroelectrodes in human epilepsy. Brain. 133 (1), 33-45 (2010).

15. Jacobs, J., LeVan, P., Chander, R., Hall, J., Dubeau, F., \& Gotman, J. Interictal high-frequency oscillations $(80-500 \mathrm{~Hz})$ are an indicator of seizure onset areas independent of spikes in the human epileptic brain. Epilepsia. 49 (11), 1893-1907 (2008).

16. Bragin, A., Mody, I., Wilson, C. L., \& Engel, J. Local generation of fast ripples in epileptic brain. J Neurosci. 22 (5), 2012-2021, (2002).

17. Worrell, G.A., \& Gardner, A.B. et al. High-frequency oscillations in human temporal lobe: simultaneous microwire and clinical macroelectrode recordings. Brain. 131 (4), 928-937 (2008).

18. Engel Jr, J., Bragin, A., Staba, R., \& Mody, I. High-frequency oscillations: What is normal and what is not? Epilepsia. 50 (4), $598-604$ (2009).

19. Traub, R. Fast Oscillations and Epilepsy. Epilepsy Curr. 3 (3), 77-79 (2003).

20. Blanco, J., \& Stead, M. et al. Unsupervised Classification of High-Frequency Oscillations in Human Neocortical Epilepsy and Control Patients. J. Neurophysiol. 104 (5), 2900-2912 (2010).

21. Rodin, E., Smid, N., \& Mason, K. The grand mal pattern of Gibbs, Gibbs and Lennox. Electroencephalogr Clin Neurophysiol. 40 (4), $401-406$ (1976). 
22. Nealis, J.G., \& Duffy, F.H. Paroxysmal beta activity in the pediatric electroencephalogram. Ann Neurol. 4 (2), 112-116 (1978).

23. Halasz, P., Terzano, M., Parrino, L., \& Bodizs, R. The nature of arousal in sleep. J. Sleep. Res. 13 (1), 1-23 (2004).

24. Kobayashi, K., \& Oka, M. et al. Very Fast Rhythmic Activity on Scalp EEG Associated with Epileptic Spasms. Epilepsia. 45 (5), $488-496$ (2004).

25. Inoue, T., Kobayashi, K., Oka, M., Yoshinaga, H., \& Ohtsuka, Y. Spectral characteristics of EEG gamma rhythms associated with epileptic spasms. Brain. Dev. 30 (5), 321-328 (2008).

26. Kobayashi, K., Jacobs, J., \& Gotman, J. Detection of changes of high-frequency activity by statistical time-frequency analysis in epileptic spikes. Clin Neurophysiol. 120 (6), 1070-1077 (2009).

27. Kobayashi, K., Watanabe, Y., Inoue, T., Oka, M., Yoshinaga, H., \& Ohtsuka, Y. Scalp-recorded high-frequency oscillations in childhood sleepinduced electrical status epilepticus. Epilepsia. 51 (10), 2190-2194 (2010).

28. Andrade-Valenca, L.P., Dubeau, F., Mari, F., Zelmann, R., \& Gotman, J. Interictal scalp fast oscillations as a marker of the seizure onset zone Neurology. 77 (6), 524-531 (2011).

29. Melani, F., Zelmann, R., Dubeau, F., \& Gotman, J. Occurrence of scalp-fast oscillations among patients with different spiking rate and their role as epileptogenicity marker. Epilepsy Res. 106 (3), 345-356 (2013).

30. Claus, S., Velis, D., Lopes da Silva, F., Viergever, M., \& Kalitzin, S. High frequency spectral components after Secobarbital: The contribution of muscular origin-A study with MEG/EEG. Epilepsy Res. 100 (1-2), 132-141 (2012).

31. Zimmermann, R., \& Scharein, E. MEG and EEG show different sensitivity to myogenic artifacts. Neurol Clin Neurophysiol. 78, (2004).

32. Papadelis, C., Poghosyan, V., Fenwick, P., \& loannides, A. MEG's ability to localise accurately weak transient neural sources. Clin Neurophysiol. 120 (11), 1958-1970 (2009).

33. Miao, A., \& Xiang, J. et al. Using ictal high-frequency oscillations $(80-500 \mathrm{~Hz})$ to localize seizure onset zones in childhood absence epilepsy: A MEG study. Neuroscience Lett. 566, 21-26 (2014).

34. Rampp, S., \& Kaltenhäuser, M. et al. MEG correlates of epileptic high gamma oscillations in invasive EEG. Epilepsia. 51 (8), $1638-1642$ (2010).

35. Tenney, J.R., \& Fujiwara, H. et al. Low- and high-frequency oscillations reveal distinct absence seizure networks. Ann Neurol. 76 (4), $558-567$ (2014).

36. Xiang, J., et al. Frequency and spatial characteristics of high-frequency neuromagnetic signals in childhood epilepsy. Epileptic Disord. 11 (2), 113-125 (2009).

37. van Klink, N., Hillebrand, A., \& Zijlmans, M. Identification of epileptic high frequency oscillations in the time domain by using MEG beamformer-based virtual sensors. Clin Neurophysiol. 127 (1), 197-208 (2016).

38. von Ellenrieder, N., \& Pellegrino, G. et al. Detection and Magnetic Source Imaging of Fast Oscillations $(40-160 \mathrm{~Hz})$ Recorded with Magnetoencephalography in Focal Epilepsy Patients. Brain Topogr. 29 (2), 218-231 (2016).

39. Rampp, S., \& Kaltenhäuser, M. et al. MEG correlates of epileptic high gamma oscillations in invasive EEG. Epilepsia. 51 (8), 1638-42. (2010).

40. Ramachandrannair R., \& Ochi, A. et al. Epileptic spasms in older pediatric patients: MEG and ictal high-frequency oscillations suggest focalonset seizures in a subset of epileptic spasms. Epilepsy Res. 78 (2-3), 216-24. (2008).

41. Bathelt, J., O'Reilly, H., de Haan, M. Cortical source analysis of high-density EEG recordings in children. $J$ Vis Exp. 88:e51705. (2014).

42. Liu, H., Tanaka, N., Stufflebeam, S., Ahlfors, S., Hämäläinen, M. Functional Mapping with Simultaneous MEG and EEG. M. J Vis Exp. 40 e1668. (2010).

43. Papadelis, C., Harini, C., Ahtam, B., Doshi, C., Grant, E., Okada, Y. Current and emerging potential for magnetoencephalography in pediatric epilepsy. J Ped Epilepsy. 2: 73-85 (2013).

44. Gaetz, W., Gordon, R.S., Papadelis, C., Fujiwara, H., Rose, D.F., Edgar, J.C., Schwartz, E.S., Roberts, T.P.L. Magnetoencephalography for Clinical Pediatrics: Recent Advances in Hardware, Methods, and Clinical Applications. J Pediatr Epilepsy. 4 (4): 139-155 (2015).

45. Prabhu, S., \& Mahomed, N. Imaging of intractable paediatric epilepsy. SA Journal of Radiology. 19 (2), 1-10 (2015).

46. Tadel, F., Baillet, S., Mosher, J.C., Pantazis, D., Leahy, R.M. Brainstorm: A User-Friendly Application for MEG/EEG Analysis. Comput Intell Neurosci. Article ID 879716 (2011).

47. Bagic, A.I., Knowlton, R.C., Rose, D.F., \& Ebersole, J.S. American Clinical Magnetoencephalography Society Clinical Practice Guideline 1 . Recording and Analysis of Spontaneous Cerebral Activity. J Clin Neurophysiol. 0 (0), 1-7 (2011).

48. Noachtar, S., Binnie, C., Ebersole, J., Mauguière, F., Sakamoto, A., \& Westmoreland, B. A glossary of terms most commonly used by clinical electroencephalographers and proposal for the report form for the EEG findings. The International Federation of Clinical Neurophysiology. Electroencephalogr Clin Neurophysiol Suppl. 52, 21-41 (1999).

49. Staba, R.J., Wilson, C.L., \& Bragin, A., et al. High-frequency oscillations recorded in human medial temporal lobe during sleep. Ann'Neurol. 56 (1), 108-115 (2004).

50. Benar, C. G., Chauviere, L., Bartolomei, F., \& Wendling, F. Pitfalls of high-pass filtering for detecting epileptic oscillations: a technical note on "false" ripples. Clin Neurophysiol. 121 (3), 301-310. (2010).

51. Crepon, B., \& Navarro, V. et al. Mapping interictal oscillations greater than $200 \mathrm{~Hz}$ recorded with intracranial macroelectrodes in human epilepsy. Brain. 133 (1), 33-45 (2010)

52. Jmail, N., \& Gavaret, M., et al. A comparison of methods for separation of transient and oscillatory signals in EEG. J Neurosci Methods. 199 (2), 273-89 (2011)

53. Wang, S., \& Wang, I.Z., et al. Ripple classification helps to localize the seizure-onset zone in neocortical epilepsy. Epilepsia. 54 (2), $370-6$ (2013).

54. Worrell, G.A., Jerbi, K., Kobayashi, K., Lina, J.M., Zelmann, R., \& Le Van Quyen, M. Recording and analysis techniques for high-frequency oscillations. Prog Neurobio. 98 (3), 265-278 (2012).

55. Gibbs, J.W. Fourier's series. Nature. 59, 606 (1899).

56. Burnos, S., \& Hilfiker, P. et al. Human Intracranial High Frequency Oscillations (HFOs) Detected by Automatic Time-Frequency Analysis. PLOS ONE. 9 (4) (2014).

57. Jacobs, J., LeVan, P., Châtillon, C., Olivier, A., Dubeau, F., \& Gotman, J. High frequency oscillations in intracranial EEGs mark epileptogenicity rather than lesion type. Brain. $132(4), 1022-1037$ (2009).

58. Urrestarazu, E., Chander, R., Dubeau, F., \& Gotman, J. Interictal high-frequency oscillations (100-500 Hz) in the intracerebral EEG of epileptic patients. Brain. 130 (9), 2354-2366 (2007).

59. Amiri, M., Lina, J.-M., Pizzo, F., \& Gotman, J. High Frequency Oscillations and spikes: Separating real HFOs from false oscillations. Clin Neurophysiol. 127 (1), 187-96 (2015). 
60. Zelmann, R., Lina, J.M., Schulze-Bonhage, A., Gotman, J., \& Jacobs, J. Scalp EEG is not a Blur: It Can See High Frequency Oscillations Although Their Generators are Small. Brain Topogr. 27 (5), 683-704 (2014).

61. Chowdhury, R.A., Lina, J.M., Kobayashi, E., \& Grova, C. MEG source localization of spatially extended generators of epileptic activity: comparing entropic and hierarchical bayesian approaches. PLoS One. 8 (2):e55969 (2013).

62. Chowdhury, R.A., Merlet, I., Birot, G., Kobayashi, E., Nica, A., Biraben, A., Wendling, F., Lina, J.M., Albera, L., \& Grova, C. Complex patterns of spatially extended generators of epileptic activity: Comparison of source localization methods cMEM and 4-ExSo-MUSIC on high resolution EEG and MEG data. Neuroimage. 143, 175-195. (2016).

63. Pellegrino, G., Hedrich, T., Chowdhury, R., Hall, J.A., Lina, J.M., Dubeau, F., Kobayashi, E., \& Grova C. Source localization of the seizure onset zone from ictal EEG/MEG data. Hum Brain Mapp. 37(7), 2528-46 (2016).

64. Grova, C., Aiguabella, M., Zelmann, R., Lina, J.M., Hall, J.A., \& Kobayashi, E. Intracranial EEG potentials estimated from MEG sources: A new approach to correlate MEG and iEEG data in epilepsy. Hum Brain Mapp. 37(5), 1661-83 (2016).

65. Lina, J.M., Chowdhury, R.A., Lemay, E., Kobayashi, E., \& Grova, C. Wavelet-based localization of oscillatory sources from magnetoencephalography data. IEEE Trans Biomed Eng. 61 (8), 2350-64 (2014).

66. Dale, A. M., Fischl, B., Sereno, M. I. Cortical surface-based analysis, I: segmentation and surface reconstruction. Neuroimage. 9, 179-194 (1999).

67. Fischl, B., Sereno, M. I., Dale, A. M. Cortical surface-based analysis, II: inflation, flattening, a surface-based coordinate system. Neuroimage. 9, 195-207 (1999).

68. Gramfort, A., Papadopoulo, T., Olivi, E., \& Clerc, M. OpenMEEG: opensource software for quasistatic bioelectromagnetics. Biomed Eng OnLine. 9 (1), 45 (2010).

69. Akiyama, T., \& McCoy, B. et al. Focal resection of fast ripples on extraoperative intracranial EEG improves seizure outcome in pediatric epilepsy. Epilepsia. 52 (10), 1802-1811 (2011).

70. Leahy, R.M., Mosher, J.C., Spencer, M.E., Huang, M.X., Lewine, J.D. A study of dipole localization accuracy for MEG and EEG using a human skull phantom. Electroencephalogr Clin Neurophysiol. 107(2), 159-73, (1989).

71. Hunold, A., Haueisen, J., Ahtam, B., Doshi, C., Harini, C., Camposano, S., Warfield, S.K., Grant, P.E., Okada, Y., \& Papadelis, C. Localization of the epileptogenic foci in tuberous sclerosis complex: a pediatric case report. Front Hum Neurosci. 8 (175) (2014).

72. Taulu, S., Kajola, M., \& Simola, J. Suppression of interference and artifacts by the signal space separation method. Brain Topogr. 16 (4), 269-275 (2004).

73. Taulu, S., \& Simola, J. Spatiotemporal signal space separation method for rejecting nearby interference in MEG measurements. Phys Med Biol. 51 (7), 1759-1768. (2006).

74. Bénar, C.G., Chauvière, L., Bartolomei, F., Wendling, F. Pitfalls of high-pass filtering for detecting epileptic oscillations: a technical note on false ripples. Clin Neurophysiol. 121 (3), 301-10. (2010).

75. Dubarry, A.S., \& Badier, J.M., et al. Simultaneous recording of MEG, EEG and intracerebral EEG during visual stimulation: from feasibility to single-trial analysis. Neuroimage. 99, 548-58. 9 (2014).

76. Chrobak, J.J., \& Buzsaki, G. High-frequency oscillations in the output of the hippocampal-entorhinal axis of the freely behaving rat. $J$ Neurosci. 19 (9), 3056-66, (1996).

77. Abend, N.S., \& Gutierrez-Colina, A. et al. Interobserver Reproducibility of Electroencephalogram Interpretation in Critically III Children. J Clin Neurophysiol. 28 (1), 15-19 (2011).

78. Benbadis, S.R., LaFrance, W.C., Papandonatos, G.D., Korabathina, K., Lin, K., \& Kraemer, H.C. Interrater reliability of EEG-video monitoring Neurology. 73 (11), 843-846 (2009).

79. von Ellenrieder, N., Andrade-Valença, L., Dubeau, F., \& Gotman, J. Automatic detection of fast oscillations (40-200Hz) in scalp EEG recordings. Clin Neurophysiol. 123 (4), 670-680 (2012).

80. Zelmann, R., Mari, F., Jacobs, J., Zijlmans, M., Dubeau, F., \& Gotman, J. A comparison between detectors of high frequency oscillations. Clin Neurophysiol. 123 (1), 106-116 (2011).

81. Birot, G., Kachenoura, A., Albera, L., Bénar, C., \& Wendling, F. Automatic detection of fast ripples. J Neurosci Methods. 213 (2), $236-249$ (2013). 